\title{
Editorial
}

\section{Special Issue on Wind Turbine Prognostics and Health Management}

\author{
David He, Eric Bechhoefer, and Abhinav Saxena
}

$\mathrm{W}$

IND POWER generating capacity was 239

GW at the end of 2011, with a further 46

GW of installed capacity to be operational by the end of 2012 . While only providing $2.8 \%$ of the energy produced in the United States, it is anticipated that by 2030 , almost $20 \%$ of the total electrical energy will come from wind. This widespread deployment of industrial wind projects will require a more proactive maintenance strategy in order to be more cost competitive with traditional energy systems, such as natural gas or coal. This will be particularly true for offshore wind projects, where availability of the site for maintenance can be restricted for extended periods of time due to weather conditions. Prognostics and Health Management (PHM) of these assets can improve operational availability while reducing the cost of unscheduled maintenance.

This Special Issue on Wind Turbine Prognostics and Health Management contains 14 excellent papers that highlight a wide range of current research and application topics related to wind turbine PHM. Fault diagnostics is an important aspect of wind turbine PHM. Eight papers included in this special issue deal with fault diagnostics of different parts of a wind turbine. Each of these papers presents different fault diagnostic techniques and sensing technologies. The paper by Waters, Beaujean, \& Vendittis presents a vibrations-based method to detect, localize, and identify a faulty bearing in an ocean turbine electric motor. In their paper, Li \& Frogley develop a vibration based method using adaptive filtering technique for fault detection in wind turbine gear transmission systems. The two papers by Godwin \& Matthews and Zhao, Siegel, Lee, \& Su deal with fault diagnosis using SCADA data. In particular, Godwin \& Matthews present a data driven classification system for the diagnosis of wind turbine pitch faults, while the paper by Zhao, Siegel, Lee, \& Su discusses the CMS and SCADA data and assesses drivetrain degradation over its lifecycle. The paper by Tamilselvan,

Wang, Sheng, \& Twomey presents a vibrationbased, two-stage fault detection framework for failure diagnosis of rotating components in wind turbines. Chase, Danai, Lackner, \& Manwell, in their paper, introduce a damage estimation method for blade and tower damage detection in operating wind turbines. In addition to the fault diagnostic methods based on vibration signals, two papers present methods developed using acoustic emission (AE) signals. The paper by Niknam, Thomas, Hines, \& Sawhney presents an AE based method for detecting a faulty bearing subject to unbalance. In the paper by Qu, Bechhoefer, He, \& Zhu, a new acoustic emission sensor based gear fault detection approach is presented. This approach combines a heterodyne based frequency reduction technique with time synchronous average and spectral kurtosis to process $\mathrm{AE}$ sensor signals and extract features as condition indictors for gearbox fault detection.

Prognostics is another important aspect of wind turbine PHM. Two papers included in this special issue specifically deal with prognostics of different components in a wind turbine. In their paper, Hussain \& Gabbar present vibration analysis and time series prediction based methods for wind turbine gearbox prognostics. In the paper by Zhu, Yoon, He, Qu, \& Bechhoefer, an online lubrication oil condition monitoring and remaining useful life prediction method using particle filtering technique and commercially available online sensors is presented. One important issue in developing wind turbine PHM systems is to develop an operational-condition-independent condition monitoring technique. The paper by Yang, Sheng, \& Court addresses this issue by developing three operational-conditionindependent criteria. One special characteristic of wind turbine PHM is that wind turbines are more likely to be subjected to considerable stresses due to unpredictable environmental conditions resulting from rapidly changing local dynamics than other types of machinery. The paper by Frost, Goebel \& Obrecht explores the integration of 
condition monitoring of wind turbine blades with contingency control to balance the trade-offs between maintaining system health and energy capture. Risk assessment represents an important step toward successful implementation of wind turbine PHM. In their paper, Dinmohammadi \& Shafiee present a fuzzy-FMEA approach for risk and failure mode analysis in offshore wind turbine systems. Life cycle cost has to be considered in developing an effective wind turbine PHM, and the paper by Lesmerises \& Crowley presents a reliability-based statistical analysis to determine which PHM strategy will yield the lowest life cycle cost for wind turbine gearboxes.

We, the editors, are confident that this special issue containing research papers on wind turbine prognostics and health management with both an academic and industrial focus will push further the wind turbine research PHM and help to bring more advanced PHM technologies into the industrial applications.
We would also like to thank the authors for their contributions and express our sincere appreciation to the reviewers for their time and expertise in providing valuable feedback.

\author{
DAVID HE, Guest Editor \\ Dept. of Mechanical and Industrial Engineering \\ University of Illinois- Chicago \\ Chicago, IL 60607 USA
}

\author{
ERIC BECHHOEFER, Guest Editor \\ Green Power Monitoring Systems, LLC \\ Burlington, VT 05401 USA
}

\author{
ABHINAV SAXENA, Editor \\ Intelligent System Division \\ NASA Ames Research Center \\ Moffett Field, CA 94035 USA
}

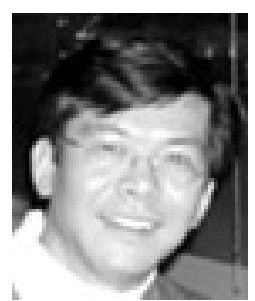

David He received his B.S. degree in metallurgical engineering from Shanghai University of Technology, China, MBA degree from The University of Northern Iowa, and Ph.D. degree in industrial engineering from The University of Iowa in 1994. Dr. He is a Professor and Director of the Intelligent Systems Modeling \& Development Laboratory in the Department of Mechanical and Industrial Engineering at The University of Illinois- Chicago. Dr. He's research areas include: machinery health monitoring, diagnosis and prognosis, complex systems failure analysis, quality and reliability engineering, and manufacturing systems design, modeling, scheduling and planning.

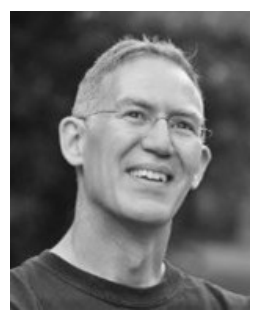

Eric Bechhoefer received his B.S. in Biology from the University of Michigan, his M.S. in Operations Research from the Naval Postgraduate School, and a Ph.D. in General Engineering from Kennedy Western University. His is a former Naval Aviator who has worked extensively on condition based maintenance, rotor track and balance, vibration analysis of rotating machinery and fault detection in electronic systems. Dr. Bechhoefer is a board member of the Prognostics Health Management Society, and a member of the IEEE Reliability Society.

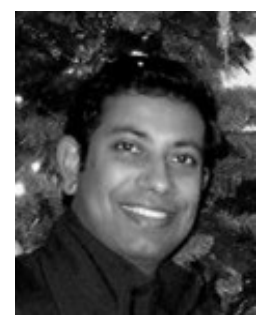

Abhinav Saxena is a Research Scientist with SGT Inc. at the Prognostics Center of Excellence NASA Ames Research Center, Moffett Field CA. His research focus lies in developing and evaluating prognostic algorithms for engineering systems. $\mathrm{He}$ is a $\mathrm{PhD}$ in Electrical and Computer Engineering from Georgia Institute of Technology, Atlanta. He earned his B.Tech in 2001 from Indian Institute of Technology (IIT) Delhi.

Dr. Saxena is a Technical Fellow for Prognostics at SGT Inc. and serving as Editor-in-Chief of International Journal of Prognostics and Health Management since 2011. 